hep-ph/0405199

\title{
Family Unification on an Orbifold
}

\author{
Zhenyu Han* and Witold Skiba ${ }^{\dagger}$ \\ Department of Physics, Yale University, New Haven, CT 06520
}

\begin{abstract}
We construct a family-unified model on a $Z_{2} \times Z_{2}$ orbifold in five dimensions. The model is based on a supersymmetric $S U(7)$ gauge theory. The gauge group is broken by orbifold boundary conditions to a product of grand unified $S U(5)$ and $S U(2) \times U(1)$ flavor symmetry. The structure of Yukawa matrices is generated by an interplay between spontaneous breaking of flavor symmetry and geometric factors arising due to field localization in the extra dimension.
\end{abstract}

\footnotetext{
* email address: zhenyu.han@yale.edu

$\dagger$ email address: witold.skiba@yale.edu
} 


\section{INTRODUCTION}

The success of gauge coupling unification in the Minimal Supersymmetric Standard Model (MSSM) suggests there exists a Grand Unified Theory (GUT). In GUTs, one generation of fermions can be incorporated in one or more representations of a simple GUT gauge group. However, GUTs do not explain why there are three different families and do not shed any light on the pattern of the observed fermion mass spectrum and mixing angles. An immediate idea is to also assign a symmetry group for the generations, the so-called flavor group. Theories incorporating this idea can be divided into two broad categories. First, theories in which the flavor group and the GUT group are orthogonal. Second, theories that unify the flavor group and the GUT group into a larger simple group. The second approach is known as family unification [1]. Within the first category, realistic theories with continuous flavor groups $S U(3), S U(2) \times U(1), U(1)$ as well as several discrete groups have been considered. Family unified models push the unification idea a step further and are esthetically more attractive. However, four-dimensional models of family unification usually suffer from the problem of mirror families, see Ref. [2] for a review.

In the last few years the notion of symmetry breaking by orbifold boundary conditions in extra dimensions has been revitalized. Orbifold breaking has been used to address various problems ranging from electroweak symmetry breaking [3], supersymmetry breaking [4] to GUT model building [5, 6]. For example, in Ref. [5] orbifold compactification is used to break the $S U(5)$ GUT group to the Standard Model group and solve the doublet-triplet splitting problem that is difficult to overcome in $4 \mathrm{D}$ models. One of the reasons we use orbifold boundary conditions is to give large masses to mirror fermions, as noticed for example in Ref. [2].

In this article, we construct a family-unified model in 5 dimensions. To maintain the unification of the gauge couplings our model incorporates supersymmetry. The fifth dimension is compactified and we impose orbifold boundary conditions on all fields propagating in the fifth dimension. The role of the boundary conditions is threefold. We use the orbifold breaking to get rid of mirror families, break family-unified gauge group to a product of GUT and flavor symmetry, and also reduce the amount of supersymmetry to $\mathcal{N}=1$ in $4 \mathrm{D}$. Given that the quarks of the third generation are a lot heavier than the quarks of the first two generations, it seems natural that the light families form a doublet, while the third family a singlet under the flavor group. Consequently, models using $S U(2) \times U(1)$ flavor symmetry are quite successful in reproducing the mass spectrum [8]. We embed the flavor $S U(2) \times U(1)$ and $S U(5)$ GUT group in an $S U(7)$ family unified gauge group. A similar setup was studied in Ref. 9], where an $S U(7)$ family unified model was considered. However, in Ref. 9] the GUT group is flipped $S U(5)$ and the emphasis is on the doublet-triplet splitting problem. A number of authors discussed flavor in extra dimensions, see Ref. 7] and references within.

The $S U(7)$ gauge group is broken by the boundary conditions to $S U(5) \times S U(2) \times$ $U(1)$. Both the $S U(5)$ and the flavor groups are broken further by expectation values of Higgs fields. The pattern of Yukawa matrices is generated by both spontaneous breaking of the flavor group and geometric factors due to field localization. Some of the fields in our model propagate in the bulk, while others are localized at the orbifold fixed points. Therefore, wavefunction overlap suppresses certain couplings with respect to others. All of the flavor physics takes place at very high energy scales, comparable to the GUT scale. Supersymmetry breaking terms are of order the electroweak scale and are irrelevant for the discussion of flavor. We will not discuss the breaking of $\mathcal{N}=1$ supersymmetry in any 
detail since this is not the focus of this paper. Any standard mechanism of communicating supersymmetry breaking in a flavor-diagonal manner could be incorporated into our model. Standard gauge mediation [10] could operate if SUSY breaking and messenger fields are localized at an orbifold fixed point. By extending the model to one more dimension one could create an appropriate setup for either anomaly mediation [11] or gaugino mediation [12] of supersymmetry breaking.

In the next section, we describe the field content and interactions needed to produce Yukawa matrices. We summarize our results in Section [II. The details concerning numerical determination of the high-energy parameters from the data are presented in Appendix B.

\section{THE MODEL}

Our model is based on a supersymmetric field theory in five dimensions. The fifth dimension is compactified on a $\left(Z_{2} \times Z_{2}\right)$ orbifold. We parameterize the fifth dimension, described by coordinate $y$, as an interval with $y \in\left[0, \frac{\pi R}{2}\right]$. This interval can be thought of as obtained from a circle $[0,2 \pi R]$ by identifying points related by reflections around two perpendicular axes. Under these reflections, $y \sim-y$ and $y \sim \pi-y$ such that the circle is equivalent to the $y \in\left[0, \frac{\pi R}{2}\right]$ interval. We denote these reflections as $P$ and $P^{\prime}$, respectively.

An arbitrary bulk field configuration can be decomposed into the eigenstates of the reflections $P$ and $P^{\prime}$. Since $P^{2}=P^{\prime 2}=1$ the eigenvalues must be \pm 1 . Of course, the eigenstates of the reflections have either the Dirichlet or Neumann boundary conditions at the end points of the interval. The Kaluza-Klein (KK) decomposition of a bulk field $\phi\left(x^{\mu}, y\right)$ into four dimensional mass eigenstates can be classified according to the two parities:

$$
\begin{aligned}
& \phi_{++}\left(x^{\mu}, y\right)=\sum_{n=0}^{\infty} \frac{1}{\sqrt{2^{\delta_{n 0}} \pi R}} \phi_{++}^{2 n}\left(x^{\mu}\right) \cos \frac{2 n y}{R}, \\
& \phi_{+-}\left(x^{\mu}, y\right)=\sum_{n=0}^{\infty} \frac{1}{\sqrt{\pi R}} \phi_{+-}^{2 n+1}\left(x^{\mu}\right) \cos \frac{(2 n+1) y}{R}, \\
& \phi_{-+}\left(x^{\mu}, y\right)=\sum_{n=0}^{\infty} \frac{1}{\sqrt{\pi R}} \phi_{-+}^{2 n+1}\left(x^{\mu}\right) \sin \frac{(2 n+1) y}{R}, \\
& \phi_{--}\left(x^{\mu}, y\right)=\sum_{n=0}^{\infty} \frac{1}{\sqrt{\pi R}} \phi_{--}^{2 n+2}\left(x^{\mu}\right) \sin \frac{(2 n+2) y}{R},
\end{aligned}
$$

where $x^{\mu}$ is the four dimensional coordinate and the subscripts refer to the parities under the $P$ and $P^{\prime}$ reflections. The five dimensional Lagrangian has simple dependence on $y$ when the fields are expressed in terms of KK states. The integral over the fifth dimension can be performed explicitly. One obtains then a four-dimensional Lagrangian describing a KK tower of four dimensional fields. The KK states specified in Eq. (11) have masses $\frac{2 n}{R}, \frac{2 n+1}{R}$, $\frac{2 n+1}{R}$, and $\frac{2 n+2}{R}$, respectively. The only massless $4 \mathrm{D}$ field is $\phi_{++}^{0}\left(x^{\mu}\right)$.

It turns out that the compactification scale in our model will be comparable to the GUT scale. The massive states will therefore be too heavy to correspond to observable states. The fields of the MSSM will come from the zero modes of the KK decomposition, as well as from brane fields localized at the endpoints of the interval. 


\section{A. Fields and interactions}

We now begin to describe our model in detail. The $5 \mathrm{D}$ bulk theory is an $\mathcal{N}=1$ SUSY theory with an $S U(7)$ gauge group. Such a theory has 8 supercharges and corresponds to $\mathcal{N}=2 \mathrm{SUSY}$ in four dimensions. However, the boundary conditions preserve only 4 supercharges, so that below the compactification scale the theory is a four dimensional $\mathcal{N}=1$ theory.

There is an arbitrary choice of how the reflection symmetry is represented in the space of gauge transformations. We choose the action of the two parities on the fundamental representation of the $S U(7)$ group to be $P=\operatorname{diag}\{1,1,1,1,1,1,1\}$ and $P^{\prime}=$ $\operatorname{diag}\{-1,-1,-1,-1,-1,1,1\}$. Consequently, an arbitrary tensor representation of $S U(7)$, $\phi_{k l \ldots}^{i j \ldots}$, transforms as

$$
\begin{aligned}
\phi_{k l \ldots}^{i j \ldots}(-y) & =\eta_{\phi} P_{i^{\prime}}^{i} P_{j^{\prime}}^{j} P_{k}^{k^{\prime}} P_{l}^{l^{\prime}} \ldots \phi_{k^{\prime} l^{\prime} \ldots}^{i^{\prime} j^{\prime} \ldots}(y), \\
\phi_{k l \ldots}^{i j \ldots}(\pi-y) & =\eta_{\phi}^{\prime} P_{i^{\prime}}^{\prime i} P_{j^{\prime}}^{\prime j} P_{k}^{\prime k^{\prime}} P_{l}^{\prime l^{\prime}} \ldots \phi_{k^{\prime} l^{\prime} \ldots}^{i^{\prime} j^{\prime} \ldots}(y),
\end{aligned}
$$

under the two parity transformations, where $\eta_{\phi}, \eta_{\phi}^{\prime}= \pm 1$ are the overall, "internal", parity eigenvalues. For a free field the parities can be chosen arbitrarily. Interaction terms correlate the parities of different fields. For example, the invariance of the supersymmetric Lagrangian imposes relations between parities of different components of superfields.

The 5D gauge multiplet contains a vector $A_{M}$, two gauginos $\lambda_{1}, \lambda_{2}$, and a real scalar $\Sigma$, all of which transform in the adjoint representation of $S U(7)$. We use the upper case Latin letters to denote 5D Lorentz indices, and the lower case Greek letters to denote 4D indices. The 5D SUSY Lagrangian is invariant under the reflections if

$$
\eta_{A_{\mu}}=-\eta_{A_{5}}=-\eta_{\Sigma}, \quad \eta_{\lambda_{1}}=-\eta_{\lambda_{2}}
$$

as well as an identical set of relations for $P^{\prime}$. We choose $\eta_{A_{\mu}}=\eta_{A_{\mu}}^{\prime}=\eta_{\lambda_{1}}=\eta_{\lambda_{1}}^{\prime}=1$.

Upon compactification, the first reflection breaks the $\mathcal{N}=15 \mathrm{D}$ SUSY to $\mathcal{N}=14 \mathrm{D}$ SUSY since both $A_{5}$ and $\lambda_{2}$ obtain large masses. Meanwhile, $A_{\mu}$ and $\lambda_{1}$ contain the zero modes that transform exactly as the $4 \mathrm{D} \mathcal{N}=1$ vector multiplet. Since we do not embed the parity transformations into the $\mathrm{R}$ symmetry $\mathcal{N}=1$ supersymmetry in $4 \mathrm{D}$ is preserved. The second reflection breaks the gauge group from $S U(7)$ to its $S U(5) \times S U(2) \times U(1)$ subgroup. More precisely, on the brane located at $y=\frac{\pi R}{2}$ the gauge group is broken, while in the bulk and on the brane $y=0$ the full symmetry remains. Besides the gauge multiplets, we put the $5 \mathrm{D}$ hypermultiplets in the bulk. Under the $S U(7)$ symmetry, the hypermultiplets transform as $\mathbf{1}+\mathbf{7}+\mathbf{3 5}+\overline{\mathbf{2 1}}$. $^{1}$ A hypermultiplet corresponds to two $4 \mathrm{D}$ chiral superfields with opposite parities $\left\{\Psi, \Psi^{c}\right\}$ :

$$
\begin{aligned}
& \eta_{\Psi}=-\eta_{\Psi^{c}} \\
& \eta_{\Psi}^{\prime}=-\eta_{\Psi^{c}}^{\prime}
\end{aligned}
$$

We choose $\eta_{\Psi}=\eta_{\Psi}^{\prime}=1$ so that all the massless fields come from $\Psi$. These massless fields can be expressed in terms of representations of the unbroken gauge groups $S U(5) \times S U(2) \times U(1)$.

${ }^{1}$ The $S U(7)$ field content coincides with an $S O(14)$ spinor $\mathbf{6 4}$ when the spinor is written in the $\mathrm{SU}(7)$ basis.

This suggests that our model may be embedded in a larger symmetry group. 
These representations are $T^{a}(\mathbf{1 0}, \mathbf{2})_{-1}, F^{a}(\overline{\mathbf{5}}, \mathbf{2})_{3}, S^{a}(\mathbf{1}, \mathbf{2})_{-5}$, and a neutral field $(\mathbf{1}, \mathbf{1})_{0}$, where $a=1,2$ is the $\mathrm{SU}(2)$ index. This set of $4 \mathrm{D}$ massless fields is free of gauge anomalies. In addition, there is no 5D anomaly either in the bulk or on the branes [13]. We interpret the $S U(5)$ as the GUT group and the $S U(2) \times U(1)$ as a flavor group. The zero modes $(\mathbf{1 0}, \mathbf{2})_{-1}$ and $(\overline{\mathbf{5}}, \mathbf{2})_{3}$ are chosen to be the light two families of fermions with their superpartners and $(\mathbf{1}, \mathbf{2})_{-5}$ might be the right handed neutrinos with their superpartners.

On the asymmetric brane we add all other superfields that are necessary to complete the MSSM spectrum and break the GUT and flavor symmetries. Since the $S U(7)$ gauge symmetry is broken on this brane, the localized fields do not need to form complete $S U(7)$ multiplets. First, we choose the third family to be $\mathrm{SU}(2)$ singlets: $T_{3}(\mathbf{1 0}, \mathbf{1})_{0}, F_{3}(\overline{\mathbf{5}}, \mathbf{1})_{0}$. Second, the SU(5) symmetry is broken by the conventional Higgses: $\Sigma(\mathbf{2 4}, \mathbf{1})_{0}, H(\mathbf{5}, \mathbf{1})_{0}$, $\bar{H}(\overline{\mathbf{5}}, \mathbf{1})_{0}, K(\mathbf{4 5}, \mathbf{1})_{0}$, and $\bar{K}(\overline{\mathbf{4 5}}, \mathbf{1})_{0}$. We need to include the 45 and $\overline{\mathbf{4 5}}$ representations to avoid the undesirable relations $m_{\mu} \simeq m_{s}$ and $m_{e} \simeq m_{d}$ at the GUT scale. In addition, we introduce the following fields to break the flavor $S U(2) \times U(1)$ symmetry: $\phi_{T, a}(\mathbf{1}, \mathbf{2})_{1}$, $\bar{\phi}_{T}^{a}(\mathbf{1}, \mathbf{2})_{-1}, \phi_{F a}(\mathbf{1}, \mathbf{2})_{-3}, \bar{\phi}_{F}^{a}(\mathbf{1}, \mathbf{2})_{3}, \psi(\mathbf{1}, \mathbf{1})_{-2}$, and $\bar{\psi}(\mathbf{1}, \mathbf{1})_{2}$. We will refer to these fields as "flavons". We summarize the light field content in Table 1.

\begin{tabular}{|c|c|c|}
\hline & bulk fileds & brane fields \\
\hline matter & $T^{a}(\mathbf{1 0}, \mathbf{2})_{-1}, F^{a}(\overline{\mathbf{5}}, \mathbf{2})_{3}, S^{a}(\mathbf{1}, \mathbf{2})_{-5}$ & $T_{3}(\mathbf{1 0}, \mathbf{1})_{0}, F_{3}(\overline{\mathbf{5}}, \mathbf{1})_{0}$ \\
\hline Higgs & & $\Sigma(\mathbf{2 4}, \mathbf{1})_{0}, H(\mathbf{5}, \mathbf{1})_{0}, \bar{H}(\overline{\mathbf{5}}, \mathbf{1})_{0}$ \\
fields & & $K(\mathbf{4 5}, \mathbf{1})_{0}, \bar{K}(\overline{\mathbf{4 5}}, \mathbf{1})_{0}$ \\
\hline flavons & & $\phi_{T, a}(\mathbf{1}, \mathbf{2})_{1}, \bar{\phi}_{T}^{a}(\mathbf{1}, \mathbf{2})_{-1}, \psi(\mathbf{1}, \mathbf{1})_{-2}, \bar{\psi}(\mathbf{1}, \mathbf{1})_{2}$ \\
& & $\phi_{F, a}(\mathbf{1}, \mathbf{2})_{-3}, \bar{\phi}_{F}^{a}(\mathbf{1}, \mathbf{2})_{3}$ \\
\hline
\end{tabular}

TABLE I: Light chiral superfields and their $S U(5) \times S U(2) \times U(1)$ charges.

Having listed the field content of our model, we now show how to obtain appropriate Yukawa couplings. Since our model is five-dimensional, the underlying field theory is nonrenormalizable and has a cutoff $\Lambda$ that is roughly two orders of magnitude larger than the compactification scale. To specify the Yukawa couplings we write the superpotential in terms of four-dimensional fields that is the brane fields and the zero modes of the bulk fields:

$$
\begin{aligned}
W= & T_{3} T_{3} H+T_{3} F_{3} \bar{H}+\frac{1}{\Lambda}\left[T_{3} T \phi_{T} H+F_{3} T \phi_{T} \bar{H}+T_{3} F \phi_{F} \bar{H}+T_{3} T \phi_{T} K\right. \\
& \left.+F_{3} T \phi_{T} \bar{K}+T_{3} F \phi_{F} \bar{K}+T F \psi \bar{H}+T F \psi \bar{K}\right]+\frac{1}{\Lambda^{2}}\left[T \phi_{T} T \phi_{T} H\right. \\
& \left.+T \phi_{T} F \phi_{F} \bar{H}+T \phi_{T} T \phi_{T} K+T \phi_{T} F \phi_{F} \bar{K}+T T \Sigma H \bar{\psi}+T T \Sigma K \bar{\psi}\right] .
\end{aligned}
$$

This superpotential is valid for a 4D theory below the compactification scale, so it is easy to keep track of dimensions of operators. We have explicitly indicated the $\frac{1}{\Lambda}$ suppression of dimension five and dimension six terms. Different terms in Eq. (5) are not related by any symmetries, so each term comes with a different coefficient. We have omitted the coefficients of operators for now. We will define and determine these coefficients in Appendix B.

In Eq. (5) we included almost all dimension five and six terms allowed by the gauge symmetries. We have omitted the couplings of $K$ and $\bar{K}$ to the third family fields $T_{3}$ and $F_{3}$. Also, the flavons $\bar{\phi}_{F}, \bar{\phi}_{T}$ do not appear in Eq. (15). Since the superpotential is not renormalized it is technically natural to exclude certain terms. However, we can assign global symmetries to our fields such that the unwanted terms in Eq. (5) are prohibited. Such 
symmetries also prevent $\bar{\phi}_{F}$ and $\bar{\phi}_{T}$ from appearing in the higher order terms, like $\frac{1}{\Lambda^{3}}, \frac{1}{\Lambda^{4}}$, etc. For any term, the addition of the $S U(5)$ adjoint $\Sigma$ is allowed by gauge symmetries. As we will show later, $\langle\Sigma\rangle / \Lambda$ is small. Thus, we also omitted terms with powers of $\Sigma$ whenever they would modify a lower order term that is more important.

For the sake of clarity, we have also omitted geometric suppression factors in Eq. (5). These factors are important for the structure of Yukawa matrices and are written explicitly in Sec. IIB. Such factors arise when bulk fields couple to brane fields because bulk fields propagate in 5D and their overlap with brane fields is small. A geometric factor is $\rho=$ $\frac{1}{\sqrt{\Lambda R \pi / 2}}$. For a given term, the number of powers of $\rho$ suppressing the term is equal to the number of bulk fields present in the term [14].

The 45 representation, $K$, and its conjugate $\bar{K}$ contain one $S U(2)$ doublet each. Together with the doublets coming from $H$ and $\bar{H}$ there would be four light doublets. We assume that one linear combination of doublets from $H$ and $K$ gets a large mass and the orthogonal linear combination remains light. The same thing takes place for $\bar{H}$ and $\bar{K}$. We outline how to realize this in Appendix A]. We denote the light mass eigenstates as $h_{u, d}$ and the heavy ones as $h_{u, d}^{\prime}$. In terms of these mass eigenstates

$$
\begin{array}{cl}
H_{u}=\sin \alpha h_{u}+\cos \alpha h_{u}^{\prime}, & K_{u}=\cos \alpha h_{u}-\sin \alpha h_{u}^{\prime}, \\
H_{d}=\sin \gamma h_{d}+\cos \gamma h_{d}^{\prime}, & K_{d}=\cos \gamma h_{d}-\sin \gamma h_{d}^{\prime},
\end{array}
$$

where $H_{u, d}$ and $K_{u, d}$ represent the $S U(2)$ doublet components of the corresponding fields. The mixing angles $\alpha$ and $\gamma$ are free parameters. For convenience, we define $v=\cot \gamma$ and $v^{\prime}=\cot \alpha$.

We assume that the flavons and $\Sigma$ get D-flat, SUSY-preserving, VEVs:

$$
\begin{gathered}
\frac{\left\langle\phi_{T}\right\rangle}{\Lambda}=\frac{\left\langle\bar{\phi}_{T}\right\rangle}{\Lambda}=\left(\begin{array}{c}
0 \\
\epsilon
\end{array}\right), \quad \frac{\left\langle\phi_{F}\right\rangle}{\Lambda}=\frac{\left\langle\bar{\phi}_{F}\right\rangle}{\Lambda}=\left(\begin{array}{c}
0 \\
\epsilon^{\prime}
\end{array}\right), \quad \frac{\langle\psi\rangle}{\Lambda}=\frac{\langle\bar{\psi}\rangle}{\Lambda}=\sigma, \\
\frac{\langle\Sigma\rangle}{\Lambda}=\operatorname{Diag}\left\{-\frac{2}{3} \delta,-\frac{2}{3} \delta,-\frac{2}{3} \delta, \delta, \delta\right\} .
\end{gathered}
$$

Supersymmetry is only broken by weak-scale soft masses. We do not specify the superpotential that produces these VEVs as it is not essential for our discussion, but it would not be difficult to do so.

\section{B. Yukawa matrices}

Using Eqs. (5) through (9) it is straightforward to write the Yukawa matrices in terms of the MSSM superfields. These couplings arise at the GUT scale after $\langle\Sigma\rangle$ breaks $S U(5)$ to the Standard Model. We denote the Yukawa matrices as $Y_{u}, Y_{d}$ and $Y_{l}$ for the up quarks, down quarks, and charged leptons, respectively. We do not consider the neutrino mass matrices or $\mathrm{CP}$ violating phases in this article. The Yukawa matrices are given by

$$
Y_{u} \sim\left(\begin{array}{ccc}
0 & \rho^{2} \sigma \delta(1+v) & 0 \\
-\rho^{2} \sigma \delta(1+v) & \rho^{2} \epsilon^{2}(1+v) & \frac{1}{2} \rho \epsilon(1+v) \\
0 & \frac{1}{2} \rho \epsilon(1+v) & 1
\end{array}\right) \sin \gamma
$$




$$
\begin{aligned}
& Y_{d} \sim\left(\begin{array}{ccc}
0 & \rho^{2} \sigma\left(1+v^{\prime}\right) & 0 \\
-\rho^{2} \sigma\left(1+v^{\prime}\right) & \rho^{2} \epsilon \epsilon^{\prime}\left(1+v^{\prime}\right) & \rho \epsilon\left(1+v^{\prime}\right) \\
0 & \rho \epsilon^{\prime}\left(1+v^{\prime}\right) & 1
\end{array}\right) \sin \alpha \\
& Y_{l} \sim\left(\begin{array}{ccc}
0 & \rho^{2} \sigma\left(1-3 v^{\prime}\right) & 0 \\
-\rho^{2} \sigma\left(1-3 v^{\prime}\right) & \rho^{2} \epsilon \epsilon^{\prime}\left(1-3 v^{\prime}\right) & \rho \epsilon\left(1-3 v^{\prime}\right) \\
0 & \rho \epsilon^{\prime}\left(1-3 v^{\prime}\right) & 1
\end{array}\right) \sin \alpha .
\end{aligned}
$$

We indicated the matrices with the proportionality sign because in the interest of clarity we omitted arbitrary coefficients that are also missing in Eq. (15). Compare Eqs. (B22)-(B4) in Appendix $\mathrm{B}$ that contain the full set of coefficients. The factors of $\frac{1}{2}$ that appear in the $(2,3)$ and $(3,2)$ elements of $Y_{u}$ arise because the terms $T_{3} T \phi_{T} H$ and $T_{3} T \phi_{T} K$ contribute to both elements.

We determine the magnitudes of the elements of the Yukawa matrices at the GUT scale by using the renormalization group equations for these matrices and comparing them with the masses and the CKM angles at the weak scale. The fitting procedure is described in Appendix B. As we will see the experimental data can be fitted quite accurately. Before we present the results let us make several comments about the matrices (10)-(12).

If $\epsilon \approx \epsilon^{\prime}$ the structure of the quark Yukawa couplings is very similar to the 4 texture zero symmetric quark mass matrices discussed in the literature. See Ref. [15] for a review. (Since the matrices discussed in Ref. 15] are symmetric the off-diagonal zeros are the same element and counted as one zero.) The only difference is that our matrices are antisymmetric in the $(1,2)$ and $(2,1)$ indices [8, 16]. The matrix for the leptons is similar to that for the down quarks. The zeros in the Yukawa matrices (10)-(12) are exact provided that $\bar{\phi}_{F}$ and $\bar{\phi}_{T}$ are absent in the superpotential in Eq. (5).

As discussed in Ref. [17], this kind of matrices can give us the approximate relations

$$
\begin{aligned}
\left|V_{u s}\right| & \approx\left|\sqrt{\frac{m_{u}}{m_{c}}}-e \sqrt{\frac{m_{d}}{m_{s}}}\right|, \\
\left|\frac{V_{t d}}{V_{t s}}\right| & \approx \sqrt{\frac{m_{d}}{m_{s}}},
\end{aligned}
$$

where $\phi$ is a CP violating phase defined in Ref. [17]. Of course, our matrices have only real elements, so the phase in the first relation is absent. In order to avoid the undesirable relation

$$
\left|\frac{V_{u b}}{V_{c b}}\right| \approx \sqrt{\frac{m_{s}}{m_{b}}}
$$

the $(2,3)$ and $(3,2)$ elements of the Yukawa matrices should be sufficiently large-much larger than $m_{s} / m_{b}$ and $m_{c} / m_{t}$ for d-Yukawa matrix and u-Yukawa matrix, respectively.

The $(2,3)$ and $(3,2)$ elements of $Y_{d}$ and $Y_{l}$ are too large to be neglected compared to the $(2,2)$ and $(3,3)$ diagonal elements. The off-diagonal elements are different for $Y_{d}$ and $Y_{l}$ : they are $\rho \epsilon\left(1+v^{\prime}\right)$ and $\rho \epsilon\left(1-3 v^{\prime}\right)$, respectively. Thus, the off-diagonal elements affect the largest eigenvalue of the matrix differently for the bottom Yukawa and the $\tau$ Yukawa. Therefore, the bottom- $\tau$ unification is not exact and the $b$ and $\tau$ masses can be fitted accurately. Similar observation was made in Ref. [18].

It is not possible to uniquely determine all the parameters in Eqs. (10)-(12) like $\epsilon, v$, etc. because there are arbitrary coefficients $a_{i}$ in front of every term, see Appendix B. 
Only certain combinations of the coefficients $a_{i}$ and other parameters appear in the Yukawa matrices. We would like all coefficients $a_{i}$ to be close to one since they are dimensionless couplings. We get the values of $a_{i}$ to be close to one by choosing the remaining parameters as follows

$$
\rho \epsilon=\frac{1}{30}, \quad \rho \epsilon^{\prime}=\frac{3}{40}, \quad \rho^{2} \sigma=\frac{3}{2000}, \quad \delta=\frac{1}{20}, \quad v^{\prime}=\frac{5}{3}, \quad v=\frac{2}{3} .
$$

We assume that $\rho \approx 0.1$ and infer the VEVs of flavons: $\epsilon \approx 0.33, \epsilon^{\prime} \approx 0.75$, and $\sigma \approx$ 0.15 . These VEVs are smaller than the cutoff $\Lambda$, but $\epsilon^{\prime}$ is quite close to 1 . Together with $\delta \approx 0.05$ and the GUT scale $M_{G U T} \sim 2.8 \times 10^{16} \mathrm{GeV}$, we get $\Lambda \sim 5.6 \times 10^{17} \mathrm{GeV}$ and $\frac{1}{R} \sim 8.8 \times 10^{15} \mathrm{GeV}$. These numbers give a resonable separation of the cutoff, the GUT, and the compactification scales [19]. However, the VEVs of the flavons are sufficiently close to the cutoff scale that higher dimensional operators may play an important role in the generation of Yukawa couplings in our model. The values of parameters in Eq. (16) correspond to $\tan \beta=47$, but we could make similar choices for other values of $\tan \beta$.

What is interesting is that the flavons' VEVs: $\epsilon, \epsilon^{\prime}, \sigma$ are of the same order. This is very different from many $4 \mathrm{D}$ models where the flavons usually obtain hierarchical VEVs in order to produce hierarchy in the Yukawa matrices. The geometric suppression factor does contribute in our model to generating small ratios.

\section{SUMMARY}

The underlying theory for our model is a 5D SUSY theory with an $S U(7)$ gauge group. Compactification of the fifth dimension on a $Z_{2} \times Z_{2}$ orbifold breaks SUSY to $\mathcal{N}=1$ in $4 \mathrm{D}$ as well as breaks $S U(7)$ to GUT $S U(5)$ times flavor $S U(2) \times U(1)$. The compactification scale is very close to the GUT scale, it is just a factor of three smaller than the GUT scale. Thus, our model is an ordinary SUSY GUT almost all the way to the GUT scale. In addition to symmetry breaking by boundary conditions we introduce two types of Higgs fields. First, standard Higgs fields that break GUT symmetry down to the Standard Model and give masses to the quarks and leptons. Second, flavon Higgs fields whose role is to completely break the flavor symmetry. The flavor symmetry is broken close to the GUT scale.

Bulk multiplets contain zero modes corresponding to the two lightest families that transform as a doublet under flavor $S U(2)$. The third family is a singlet under the flavor symmetry and it is localized at one of the orbifold fixed points. The $S U(7)$ gauge symmetry is not preserved at the fixed point where the third family is localized. Therefore, the third family does not come from a complete $S U(7)$ multiplet and is a flavor singlet. As far as the flavor symmetry and the light fields are concerned our model is very similar to the $4 \mathrm{D}$ model described in Ref. [8].

Our main goal was constructing a realistic pattern of Yukawa matrices at the GUT scale. We were only concerned with the quark and charged lepton sectors and completely neglected the neutrino sector. The Yukawa couplings come from the superpotential in Eq. (5), which we chose to resemble the "four zeros" texture described in Ref. [15]. The resulting Yukawa matrices, omitting a number of dimensionless constants of order one, are given in Eqs. (10), (11), and (12).

The orders of magnitude of different elements of the Yukawa matrices are governed by three different effects. The first effect is the geometry of our model. The couplings that involve both localized fields and bulk fields are suppressed due to small wavefunction overlap 
between such fields. Second, the $S U(2) \times U(1)$ flavor symmetry is broken by three different flavons and their conjugates. Among the three flavons there are two $S U(2)$ doublets and one singlets. All flavons are charged under the $U(1)$. We do not count separately the conjugates of the flavons because the VEVs of flavons with the conjugate quantum numbers are identical to maintain SUSY above the weak scale. Third, the up and down sectors are distinguished by the mixing of the Higgs doublets that come both from the $\mathbf{5}$ and the $\mathbf{4 5}$. The light up and down Higgs doublets come from different linear combinations of $\mathbf{5}$ and $\mathbf{4 5}$. Of course, any value of $\tan \beta$ other than 1 also differentiates the up and down sectors.

Our model has too many free parameters to be predictive. What we accomplished, however, is generating the Yukawa matrices in terms of a few small parameters: flavon VEVs, defined in Eqs. (8) and (16), and the geometric suppression factor. By matching to the observed fermion mass spectrum and quark mixing angles we determined the 13 nonzero parameters in the Yuklawa matrices, see Eqs. (B5)-(B7). We chose the undetermined parameters such that the dimensionless couplings are close to one.

What is interesting is that given a few arbitrary choices all dimensionless coefficients are of order one. Moreover, many of the coefficients listed in Eq. (B11) are very close to one. All the large ratios are determined in terms of the geometric suppression factor and a few flavon VEVs that are of the same order of magnitude. Obviously, a more fundamental and predictive structure of flavor is still missing. However, it is conceivable that the flavor could be generated from an interplay between geometry and flavor symmetries.

\section{Acknowledgments}

We thank M. Piai for discussions and comments on the manuscript. This research was supported in part by the US Department of Energy under grant DE-FG02-92ER-40704. WS is also supported in part by the DOE OJI program.

\section{APPENDIX A: MIXING OF HIGGS DOUBLETS}

We briefly comment on the mixing of the Higgs doublets coming from the $\mathbf{4 5}$ and $\mathbf{5}$ representations and their conjugates. As we explained in Sec. III we need the $\mathbf{4 5}$ and $\overline{\mathbf{4 5}}$ representations to avoid the equality of the lepton-down quark Yukawa couplings in the two light families. The problem is similar to $S O(10)$ unification, where one needs to introduce larger Higgs representation in addition to the 10-dimensional Higgs to incorporate realistic Yukawa couplings. The additional Higgs fields, for example $\overline{\mathbf{1 2 6}}$, would produce too many light doublets. A simple solution was presented in Ref. [20]. Similar solution works in the $S U(5)$ case and we outline it here for completeness.

We supplement the Higgs fields $H, \bar{H}, K$, and $\bar{K}$ introduced already by another pair of 45 and $\overline{\mathbf{4 5}}$ Higgs fields. Let us refer to the new fields as $K_{1}$ and $\bar{K}_{1}$. We assume that the superpotential for these Higgs fields is given by

$$
W_{\text {Higgs }}=\mu H \bar{H}+H \Sigma \bar{H}+H \Sigma \bar{K}_{1}+\bar{H} \Sigma K_{1}+M_{1} K_{1} \bar{K}+M_{2} K \bar{K}_{1} .
$$

In the equation above $\Sigma$ is the $S U(5)$ adjoint field that develops an $S U(5)$ breaking VEV given by Eq. (9) and $\langle\Sigma\rangle \propto \delta \Lambda . \quad M_{1}$ and $M_{2}$ are arbitrary mass parameters that are comparable to the GUT scale. We also assume that $\mu \approx-\delta \Lambda$ so that the $S U(2)$ doublets in $H$ 
and $\bar{H}$ are light. The mass matrix for the doublets arising from $H, \bar{H}, K, \bar{K}, K_{1}$, and $\bar{K}_{1}$ has the following structure

$$
\mathcal{M}=\left(\begin{array}{c}
H_{d} \\
K_{1 d} \\
K_{d}
\end{array}\right)^{T}\left(\begin{array}{ccc}
0 & \delta \Lambda & 0 \\
\delta \Lambda & 0 & M_{2} \\
0 & M_{1} & 0
\end{array}\right)\left(\begin{array}{c}
H_{u} \\
K_{1 u} \\
K_{u}
\end{array}\right)
$$

where we assumed that $\mu+\delta \Lambda=0$. The light eigenvalues of this mass matrix are two doublets

$$
\begin{aligned}
h_{u} & =\cos \alpha H_{u}+\sin \alpha K_{u}, \\
h_{d} & =\cos \gamma H_{d}+\sin \gamma K_{d},
\end{aligned}
$$

where $\tan \alpha=\delta \Lambda / M_{2}$ and $\tan \gamma=\delta \Lambda / M_{1}$. There is no reason to assume that $M_{1}$ and $M_{2}$ are equal, so the mixing angles of the up and down Higgs doublets are, in general, different. Clearly, the remaining Higgs doublets have masses of order the unification scale and so do other components of $K, \bar{K}, K_{1}$, and $\bar{K}_{1}$.

\section{APPENDIX B: FITTING TO THE DATA}

We now describe our procedure for determining the Yukawa matrices at the GUT scale. As we mentioned in Sec. [IA, there are no relations between the various terms in Eq. (5) since different terms are not related by symmetries to one another. Therefore, one needs to include arbitrary coefficients of order one in front of every term. After including the missing coefficients Eq. (5) becomes:

$$
\begin{aligned}
W= & a_{1} T_{3} T_{3} H+a_{2} T_{3} F_{3} \bar{H}+\frac{1}{\Lambda}\left[a_{3} T_{3} T \phi_{T} H+a_{4} F_{3} T \phi_{T} \bar{H}+a_{5} T_{3} F \phi_{F} \bar{H}+a_{6} T_{3} T \phi_{T} K\right. \\
& \left.+a_{7} F_{3} T \phi_{T} \bar{K}+a_{8} T_{3} F \phi_{F} \bar{K}+a_{9} T F \psi \bar{H}+a_{10} T F \psi \bar{K}\right]+\frac{1}{\Lambda^{2}}\left[a_{11} T \phi_{T} T \phi_{T} H\right. \\
& \left.+a_{12} T \phi_{T} F \phi_{F} \bar{H}+a_{13} T \phi_{T} T \phi_{T} K+a_{14} T \phi_{T} F \phi_{F} \bar{K}+a_{15} T T \Sigma H \bar{\psi}+a_{16} T T \Sigma K \bar{\psi}\right] .
\end{aligned}
$$

The corresponding Yukawa matrices are then

$$
\begin{aligned}
& Y_{u}=\left(\begin{array}{ccc}
0 & \rho^{2} \sigma \delta\left(a_{15}+a_{16} v\right) & 0 \\
-\rho^{2} \sigma \delta\left(a_{15}+a_{16} v\right) & \rho^{2} \epsilon^{2}\left(a_{11}+a_{13} v\right) & \frac{\rho \epsilon}{2}\left(a_{3}+a_{6} v\right) \\
0 & \frac{\rho \epsilon}{2}\left(a_{3}+a_{6} v\right) & a_{1}
\end{array}\right) \sin \gamma \\
& Y_{d}=\left(\begin{array}{ccc}
0 & \rho^{2} \sigma\left(a_{9}+a_{10} v^{\prime}\right) & 0 \\
-\rho^{2} \sigma\left(a_{9}+a_{10} v^{\prime}\right) & \rho^{2} \epsilon \epsilon^{\prime}\left(a_{12}+a_{14} v^{\prime}\right) & \rho \epsilon\left(a_{4}+a_{7} v^{\prime}\right) \\
0 & \rho \epsilon^{\prime}\left(a_{5}+a_{8} v^{\prime}\right) & a_{2}
\end{array}\right) \sin \alpha, \\
& Y_{l}=\left(\begin{array}{ccc}
0 & \rho^{2} \sigma\left(a_{9}-3 a_{10} v^{\prime}\right) & 0 \\
-\rho^{2} \sigma\left(a_{9}-3 a_{10} v^{\prime}\right) & \rho^{2} \epsilon \epsilon^{\prime}\left(a_{12}-3 a_{14} v^{\prime}\right) & \rho \epsilon\left(a_{4}-3 a_{7} v^{\prime}\right) \\
0 & \rho \epsilon^{\prime}\left(a_{5}-3 a_{8} v^{\prime}\right) & a_{2}
\end{array}\right) \sin \alpha .
\end{aligned}
$$

The Yukawa matrices are defined in terms of 16 coefficients $a_{i}, 3$ flavon VEVs, $\Sigma$ VEV, geometric factor $\rho$, and two Higgs mixing angles: a total of 23 parameters. However, several 
of our parameters only appear in particular combinations, which allows us to eliminate the "unobservable" combinations:

$$
\begin{aligned}
Y_{u} & =\left(\begin{array}{ccc}
0 & c_{1} & 0 \\
-c_{1} & c_{2} & c_{3} \\
0 & c_{3} & 1
\end{array}\right) \eta \\
Y_{d} & =\left(\begin{array}{ccc}
0 & c_{4} & 0 \\
-c_{4} & c_{5} & c_{6} \\
0 & c_{7} & 1
\end{array}\right) \zeta \\
Y_{l} & =\left(\begin{array}{ccc}
0 & c_{8} & 0 \\
-c_{8} & c_{9} & c_{10} \\
0 & c_{11} & 1
\end{array}\right) \zeta
\end{aligned}
$$

We are left with 13 parameters: $c_{1}$ through $c_{11}, \eta$, and $\zeta$. The experimental data, gives nine masses and three real angles in the CKM matrix. Including complex phases in our Yukawa matrices would introduce too many free parametres, so we omit the phases. If we performed a fit with the phases present the values of the real parameters might change slightly, but such change would not affect the structure of the Yukawa matrices.

To obtain the GUT scale values we use the following fermion parameters and the gauge couplings at the scale $M_{Z}[21]$ as inputs:

$$
\begin{gathered}
\alpha_{1}=0.016829, \alpha_{2}=0.033493, \alpha_{3}=0.118 \\
m_{u}=2.33 \pm 0.435 \mathrm{MeV}, m_{c}=0.677 \pm 0.0585 \mathrm{GeV}, m_{t}=181 \pm 13 \mathrm{GeV} \\
m_{d}=4.36 \pm 1.13 \mathrm{MeV}, m_{s}=72 \pm 23 \mathrm{MeV}, m_{b}=3.00 \pm 0.1 \mathrm{GeV} \\
m_{e}=486.84727 \pm 0.00014 \mathrm{keV}, m_{\mu}=102.75138 \pm 0.00033 \mathrm{MeV}, m_{\tau}=1.74669 \pm 0.000285 \mathrm{GeV} \\
V_{u s}=0.2205 \pm 0.0018, V_{c b}=0.0373 \pm 0.0018,\left|V_{u b} / V_{c b}\right|=0.08 \pm 0.02 .
\end{gathered}
$$

Given the structure of Yukawa matrices described in Eqs. (B5)-(B7) at the GUT scale, we use the one loop renormalization group equations in the MSSM 22] to compare with the weak scale data. We set the GUT scale to be $M_{G U T}=2.80 \times 10^{16}$ where the three gauge coupling constants unify. The one-loop running of the gauge couplings does not involve the Yukawa couplings, so the gauge couplings are determined at all scales before fitting the Yukawa matrices. We neglect the fact that the first few KK modes appear below $M_{G U T}$ since $R M_{G U T} \approx 3.2$. Since the logarithm of $R M_{G U T}$ is small we can neglect the effects of the KK modes below $M_{G U T}$ and we use 4D RGE equations.

In practice, we numerically evaluate the RGE equations from the GUT scale down to the weak scale. We then compare the results of the RGE running with the data and evaluate the $\chi^{2}$ using the experimental errors. The errors are severely underestimated this way because threshold corrections and two-loop effects are much larger than the experimental uncertainty of the lepton masses. However, since we have more parameters than the number of inputs we are able to get a good fit. For example, we present the numerical fit for $\tan \beta=47$ below.

$$
Y_{u}=\left(\begin{array}{ccc}
0 & -0.0001050 & 0 \\
0.0001050 & 0.005335 & 0.05848 \\
0 & 0.05848 & 1
\end{array}\right) 1.053
$$




$$
\begin{aligned}
Y_{d} & =\left(\begin{array}{ccc}
0 & 0.004744 & 0 \\
-0.004744 & 0.006898 & 0.1009 \\
0 & 0.2205 & 1
\end{array}\right) 0.4597 \\
Y_{l} & =\left(\begin{array}{ccc}
0 & -0.003774 & 0 \\
0.003774 & -0.007916 & -0.1526 \\
0 & -0.3161 & 1
\end{array}\right) 0.4597 .
\end{aligned}
$$

The total $\chi^{2}$ for this fit is 4.654 . The $\chi^{2}$ is dominated by the errors from $m_{d}$ and $m_{\tau}$, but none of the two masses with the poorest fit deviates by more than $1.5 \sigma$ from the experimental value.

To extract the physical parameters $\rho, \sigma$ and so on, we need to remember that the coefficients $a_{1}, \ldots, a_{16}$ are close to 1 . A choice of parameters is given in Eq. (16) and the corresponding coefficients are

$$
\begin{gathered}
a_{1}=1.266, \quad a_{2}=0.8935, \quad a_{3}=3, \quad a_{4}=1.006, \quad a_{5}=1.029, \quad a_{6}=2.162, \\
a_{7}=1.019, \quad a_{8}=0.9590, \quad a_{9}=1.557, \quad a_{10}=0.7611, \quad a_{11}=3, \quad a_{12}=1.141, \\
a_{13}=4.618, \quad a_{14}=0.7942, \quad a_{15}=1, \quad a_{16}=1.160 .
\end{gathered}
$$

Note that the coefficients $a_{3}, a_{11}$ and $a_{15}$ in $Y_{u}$ are set by hand. There are too many free parameters to be uniquely determined from the 13 parameters in Eqs. (B5)-(B7), so we need to arbitrarily choose some of them.

Most coefficients $a_{i}$ are very close to 1 and certainly none of the coefficients deviate from one by an order of magnitude. There are many small additional contributions to this result that we neglected, for example higher-dimensional operators, threshold corrections, higherloop effects. Since the coefficients $a_{i}$ are so close to multiples of 1 it is possible that the deviations could be accounted for by higher-order effects we neglected. This suggests that there could be a simple set of hidden symmetries responsible for this result. It certainly would be an exciting possibility.

[1] H. Georgi, Nucl. Phys. B 156, 126 (1979); F. Wilczek and A. Zee, Phys. Rev. D 25, 553 (1982).

[2] K. S. Babu, S. M. Barr, B. Kyae, Phys. Rev. D 65, 115008 (2002) arXiv:hep-ph/0202178.

[3] R. Barbieri, L. J. Hall and Y. Nomura, Phys. Rev. D 63, 105007 (2001) arXiv:hep-ph/0011311; N. Arkani-Hamed, L. J. Hall, Y. Nomura, D. R. Smith and N. Weiner, Nucl. Phys. B 605, 81 (2001) arXiv:hep-ph/0102090.

[4] T. Gherghetta and A. Pomarol, Nucl. Phys. B 602, 3 (2001) arXiv:hep-ph/0012378; M. Chaichian, A. B. Kobakhidze and M. Tsulaia, Phys. Lett. B 505, 222 (2001) arXiv:hep-th/0101210; A. Delgado and M. Quiros, Nucl. Phys. B 607, 99 (2001) arXiv:hep-ph/0103058.

[5] Y. Kawamura, Prog. Theor. Phys. 105, 999 (2001) arXiv:hep-ph/0012125; G. Altarelli and F. Feruglio, Phys. Lett. B 511, 257 (2001) arXiv:hep-ph/0102301; L. J. Hall and Y. Nomura, Phys. Rev. D 64, 055003 (2001) arXiv:hep-ph/0103125.

[6] L. J. Hall and Y. Nomura, Phys. Rev. D 65, 125012 (2002) arXiv:hep-ph/0111068; Phys. Rev. D 66, 075004 (2002) arXiv:hep-ph/0205067; A. Hebecker and J. March-Russell, Nucl. Phys. B 
613, 3 (2001) arXiv:hep-ph/0106166; Nucl. Phys. B 625, 128 (2002) arXiv:hep-ph/0107039; L. J. Hall, Y. Nomura, T. Okui and D. R. Smith, Phys. Rev. D 65, 035008 (2002) arXiv:hep-ph/0108071.

[7] K. S. Choi and J. E. Kim, Phys. Lett. B 552, 81 (2003) arXiv:hep-th/0206099; A. Aranda and J. L. Diaz-Cruz, arXiv:hep-ph/0207059 R. Kitano and T. j. Li, Phys. Rev. D 67, 116004 (2003) arXiv:hep-ph/0302073 ; C. Biggio, F. Feruglio, I. Masina and M. Perez-Victoria, Nucl. Phys. B 677, 451 (2004) arXiv:hep-ph/0305129]; I. Gogoladze, Y. Mimura and S. Nandi, Phys. Rev. D 69, 075006 (2004) arXiv:hep-ph/0311127.

[8] R. Barbieri, G. R. Dvali and L. J. Hall, Phys. Lett. B 377, 76 (1996) arXiv:hep-ph/9512388; R. Barbieri, L. J. Hall, S. Raby and A. Romanino, Nucl. Phys. B 493, 3 (1997) arXiv:hep-ph/9610449; R. Barbieri, L. J. Hall and A. Romanino, Phys. Lett. B 401, 47 (1997) arXiv:hep-ph/9702315; R. Barbieri, L. Giusti, L. J. Hall and A. Romanino, Nucl. Phys. B 550, 32 (1999) arXiv:hep-ph/9812239.

[9] K. w. Hwang and J. E. Kim, Phys. Lett. B 540, 289 (2002) arXiv:hep-ph/0205093.

[10] M. Dine, A. E. Nelson and Y. Shirman, Phys. Rev. D 51, 1362 (1995), arXiv:hep-ph/9408384; M. Dine, A. E. Nelson, Y. Nir and Y. Shirman, Phys. Rev. D 53, 2658 (1996), arXiv:hep-ph/9507378.

[11] L. Randall and R. Sundrum, Nucl. Phys. B 557, 79 (1999), arXiv:hep-th/9810155; G. F. Giudice, M. A. Luty, H. Murayama and R. Rattazzi, JHEP 9812, 027 (1998) arXiv:hep-ph/9810442.

[12] D. E. Kaplan, G. D. Kribs and M. Schmaltz, Phys. Rev. D 62, 035010 (2000), arXiv:hep-ph/9911293 ; Z. Chacko, M. A. Luty, A. E. Nelson and E. Ponton, JHEP 0001, 003 (2000), arXiv:hep-ph/9911323; M. Schmaltz and W. Skiba, Phys. Rev. D 62, 095005 (2000), arXiv:hep-ph/0001172.

[13] N. Arkani-Hamed, A. G. Cohen and H. Georgi, Phys. Lett. B 516, 395 (2001) arXiv:hep-th/0103135; C. A. Scrucca, M. Serone, L. Silvestrini and F. Zwirner, Phys. Lett. B 525, 169 (2002) arXiv:hep-th/0110073; L. Pilo and A. Riotto, Phys. Lett. B 546, 135 (2002) arXiv:hep-th/0202144; H. D. Kim, J. E. Kim and H. M. Lee, JHEP 0206, 048 (2002) arXiv:hep-th/0204132.

[14] L. Hall, J. March-Russell, T. Okui and D. R. Smith, arXiv:hep-ph/0108161

[15] H. Fritzsch and Z. z. Xing, Prog. Part. Nucl. Phys. 45, 1 (2000) arXiv:hep-ph/9912358.

[16] Z. Berezhiani and A. Rossi, JHEP 9903, 002 (1999) arXiv:hep-ph/9811447.

[17] H. Fritzsch and Z. z. Xing, Phys. Lett. B 555, 63 (2003) arXiv:hep-ph/0212195.

[18] S. M. Barr and I. Dorsner, Phys. Lett. B 556, 185 (2003) arXiv:hep-ph/0211346.

[19] A. Hebecker and J. March-Russell, Phys. Lett. B 541, 338 (2002) arXiv:hep-ph/0205143.

[20] D. G. Lee and R. N. Mohapatra, Phys. Rev. D 51, 1353 (1995) arXiv:hep-ph/9406328.

[21] H. Fusaoka and Y. Koide, Phys. Rev. D 57, 3986 (1998) arXiv:hep-ph/9712201.

[22] S. P. Martin and M. T. Vaughn, Phys. Rev. D 50, 2282 (1994) arXiv:hep-ph/9311340. 